\title{
Automatic detection of crop rows in maize fields with high weeds pressure
}

\author{
$\begin{array}{llllll}\text { M. Montalvo G. Pajares } & \text { J.M. Guerrero } & \text { J. Romeo } & \text { M. Guijarro } & \text { A. Ribeiro J.J. Ruz J.M. Cruz }\end{array}$
}

\begin{abstract}
A B S T R A C T
This paper proposes a new method, oriented to crop row detection in images from maize fields with high weed pressure. The vision system is designed to be installed onboard a mobile agricultural vehicle, i.e. submitted to gyros, vibrations and undesired movements. The images are captured under image perspective, being affected by the above undesired effects. The image processing consists of three main processes: image segmentation, double thresholding, based on the Otsu's method, and crop row detection. Image segmentation is based on the application of a vegetation index, the double thresholding achieves the separation between weeds and crops and the crop row detection applies least squares linear regression for line adjustment. Crop and weed separation becomes effective and the crop row detection can be favorably compared against the classical approach based on the Hough transform. Both gain effectiveness and accuracy thanks to the double thresholding that makes the main finding of the paper.
\end{abstract}

\section{Introduction}

\subsection{Problem statement}

The increasing development of robotics equipped with machine vision sensors applied to precision agriculture (PA) is demanding solutions for several problems. The robot navigates and acts over a site-specific area of a larger farm (Davies, Casady, \& Massey, 1998), where one important part of the information is supplied by the vision system.

An important issue related with the application of machine vision methods is that concerning the crop row and weed detection, which has attracted numerous studies in this area (Burgos-Artizzu, Ribeiro, Tellaeche, Pajares, \& Fernández-Quintanilla, 2009; Onyango \& Marchant, 2003; Sainz-Costa, Ribeiro, Burgos-Artizzu, Guijarro, \& Pajares, 2011; Tellaeche, Burgos-Artizzu, Pajares, \& Ribeiro, 2008b; Tellaeche, Burgos-Artizzu, Pajares, Ribeiro, \& FernándezQuintanilla, 2008a). This will allow site-specific treatments trying to eliminate weeds and to favor the growth of crops. The problem becomes highly complex when high weed pressure exists.

The vision system consists of a CCD-based calibrated camera with known intrinsic parameters, i.e. focal length, radial lens distortion, CCD sensor sizes and pixel resolutions. The camera is located in front of the agricultural vehicle, inclined with a tilt angle (pitch) and at a high from the ground. Yaw and roll angles are also known. This allows determining the rotation and translation matrices defining the extrinsic parameters. Thus, areas in the field can be identified onto the image plane and vice-versa. This means that given any element in the image we can determine its relative positioning on the field.

The vehicle navigates on a real terrain presenting irregularities and roughness where the spacing of crop rows is known. This produces vibrations and also swinging in the pitch, yaw and roll angles. Because of the above, the crop rows are not projected onto the image at the expected locations according to the system geometry. On the other hand the discrimination of crops and weeds in the image is a very difficult task because their Red, Green and Blue spectral components display similar values. Thus, high weed pressure, similar spectral components and unexpected projections, all together make the crop row detection a complex problem that is specifically addressed in this paper, making the main contribution.

Thus, the problem is to locate accurately the crop rows in images of maize fields with high weed pressure. These images are captured by a camera aboard a vehicle in movement. To achieve this goal, we propose a new strategy that makes use of pervious knowledge: (a) the specific arrangement of crops (maize) in the field and (b) image perspective projection and system geometry. We apply an automatic image segmentation process for greenness extraction and a double thresholding, based on the Otsu's method. After this, a total least-squares linear regression approach allows the automatic adjusting of the corresponding straight line equation to each crop row.

Crop row location has been considered already on the literature, the method proposed in this paper gains advantage over existing approaches because it has been designed to achieve high 
effectiveness in real-time applications in PA for maize fields with high weed pressure.

\subsection{Revision of methods}

Several strategies have been proposed for crop row detection. Fontaine and Crowe (2006) tested the abilities of fourth line detection algorithms to determine the position and the angle of the camera with respect to a set of artificial rows with and without simulated weeds. These were stripe analysis, blob analysis, linear regression and Hough transform. Below is a revision of methods grouped into eight categories.

(1) Methods based on the exploration of horizontal strips: Søgaard and Olsen (2003) apply RGB color image transformation to grayscale. This is done by first dividing the color image into its red, green and blue channels and then by applying the well-tested methods to extract living plant tissue described in Woebbecke, Meyer, von Bargen, and Mortensen (1995). After this, the gray-scale image is divided into horizontal strips where maximum gray values indicate the presence of a candidate row, each maximum determines a row segment and the center of gravity of the segment is marked at this strip position. Crop rows are identified by joining marked points through a similar method to the one utilized in the Hough transform or by applying linear regression. Sainz-Costa et al. (2011) have developed a strategy based on analysis of video sequences for identifying crop rows. Crop rows persist along the directions defined by the perspective projection with respect the $3 \mathrm{D}$ scene in the field. Exploiting this fact, they apply gray scale transformation based on the approach proposed by Ribeiro, FernándezQuintanilla, Barroso, and García-Alegre (2005) and then the image is binarized applying a thresholding technique. Each image is divided into four horizontal strips. Rectangular patches are drawn over the binary image to identify patches of crops and rows. The gravity centers of these patches are used as the points defining the crop rows and a line is adjusted considering these points. The first frame in the sequence is used as a lookup table that guides the full process for determining positions where the next patches in subsequent frames are to be identified. Hague, Tillet, and Wheeler (2006) transform the original RGB image to gray scale. The transformed image is then divided into eight horizontal bands. The intensity of the pixels across these bands exhibit a periodic variation, due to the parallel crop rows. Since the camera characteristics, pose and the crop row spacing are known a priori, the row spacing in image pixels can be calculated for each of the horizontal bands using a pinhole model of the camera optics. A band-pass filter can then be constructed which will enhance this pattern, and has a given frequency domain response. In images with high weed pressure, weeds and crop appear together forming a unique patch and gravity centers and other strip features are incorrectly identified.

(2) Methods based on the Hough transformation: According to Slaughter, Giles, and Downey (2008), one of the most commonly used machine vision methods for identifying crop rows is based upon the Hough (1962) transform. It was intended to deal with discontinuous lines, where the crop stand is incomplete with gaps in crop rows due to poor germination or other factors that result in missing crop plants in the row. It has been intended for real-time automatic guidance of agricultural vehicles (Astrand \& Baerveldt, 2005; Hague, Marchant, \& Tillett, 1997; Leemans \& Destain, 2006; Marchant, 1996). It is applied to binary images, which are obtained by applying similar technique to the ones explained above, i.e. RGB image transformation to grayscale and binzarization (Tellaeche, Pajares, Burgos-Artizzu, \& Ribeiro, 2011; Tellaeche et al., 2008a, 2008b). Gée, Bossu, Jones, and Truchetet (2008) apply a double Hough transform under the assumption that crop rows are the only lines of the image converging to the vanishing point, the remainder lines are rejected, additional constraints such as inter-row spacing and perspective geometry concepts help to identify the lines. It is required to determine the threshold required by the Hough transform to determine maximum peaks values (Jones, Gée, \& Truchetet, 2009a, 2009b) or predominant peaks (Rovira-Más, Zhang, Reid, \& Will 2005). Depending on the crop densities several lines could be feasible and a posterior merging process is applied to lines with similar parameters (Tellaeche et al., 2008a, 2008b, 2011). Ji and Qi (2011) report that Hough transform is slow due to the huge computation, they propose a randomized Hough transform to reduce computational time. Some modifications have been proposed to improve the Hough transformation such as the one proposed in Asif, Amir, Israr, and Faraz (2010), which is applied only to those points which are edge points along the crops. But this requires the application of techniques for edge extraction. Also the randomized Hough transformation has been proposed with this goal (Ji \& Qi, 2011). It is intended to avoid redundant computations in the Hough transform. It operates iteratively by randomly sampling a set of points to compute a single localization in the Hough space. Since two pixels are trivially collinear, the parameters of the line on which they lie can be estimated. These parameters are used to increment the accumulator cell in the Hough space. The Hough transform is computationally expensive and the randomized Hough transform requires selecting pairs of points to be considered as forming a line, i.e. pairs of points belonging to a crop row. This becomes unfeasible in images with a high weed pressure because crop rows are often indistinguishable and pairs of points cannot be identified as belonging to a given crop row.

(3) Vanishing point-based: Pla, Sanchiz, Marchant, and Brivot (1997) propose an approach which identifies regions (crops/weeds and soil) by applying color image segmentation. They use the skeleton of each defined region as a feature to work out the lines which define the crop. The resulting skeletons of each region can be used as curves which define the underlying structure of the crop and to extract the straight lines where the plants and soil rows lie. Segments in the skeletons are defined as chains of connected contour points and they must be of a defined length. This allows selecting candidate lines that meet the vanishing point for crop row detection. The vanishing point is detected using previous information about the vanishing point found in the previous images, performing a sort tracking on the vanishing point. This process is highly dependent of skeletons, which are not always easy to extract, particularly in images with a high weed pressure.

(4) Methods based on linear regression: Some of the techniques above apply this approach. Billingsley and Schoenfisch (1997) reported a crop detection system that is relatively insensitive to additional visual 'noise' from weeds. They used linear regression in each of three crop row segments and a cost function analogous to the moment of the bestfit line to detect lines fitted to outliers (i.e., noise and weeds) as a means of identifying row guidance information. As mentioned above, Søgaard and Olsen (2003) also apply linear regression. A feasible approach applicable 
when pixels belonging to crop rows are well separated from weeds. Its effectiveness is highly affected by pixels belonging to weeds. Therefore, linear regression is only feasible if weeds and crops have been separated. In our proposed approach we separate weed and crops thank to the double Otsu's approach, making the linear regression a feasible strategy.

(5) Stereo-based approach: Kise, Zhang, and Rovira-Más (2005) or Kise and Zhang (2008) developed a stereovision-based agricultural machinery crop-row tracking navigation system. Stereo-image processing is used to determine 3D locations of the scene points of the objects of interest from the obtained stereo image. Those 3D positions, determined by means of stereo image disparity computation, provide the base information to create an elevation map which uses a 2D array with varying intensity to indicate the height of the crop. This approach requires crops with significant heights with respect the ground. Because in maize fields during the treatment stage the heights are not relevant, this approach becomes ineffective in our application. RoviraMás, Zhang, and Reid (2008) have applied and extended stereovision techniques to other areas inside PA. As before, only feasible if crops or weeds in the 3D scene display relevant heights and these heights are different in crops and weeds.

(6) Methods based on blob analysis: This method finds and characterizes regions of contiguous pixels of the same value in a binarized image (Fontaine \& Crowe, 2006). The algorithm searches for white blobs (inter-row spaces) of more than 200 pixels, as smaller blobs could represent noise in the crop rows. Once the blobs were identified, the algorithm determines the angle of their principal axes and the location of their center of gravity. For a perfectly straight white stripe, the center of gravity of the blob was over the centerline of the white stripe, and the angle was representative of the angle of the inter-row spaces. The algorithm returned the angle and center of gravity of the blob closest to the center of the image. Identification of blobs in images infested with high weeds pressure becomes unfeasible.

(7) Methods based on the accumulation of green plants: Olsen (1995) proposed a method based on the consideration that along the crop row appear an important accumulation of green parts in the image. The image is grayscale transformed where green parts appear clearer that the rest. A sum-curve of gray levels is obtained for a given rectangular region exploring all columns in the rectangle. It is assumed that vertical lines follow this direction in the image. Obviously, the images are free of perspective projection because they are acquired with the camera in orthogonal position. A sinusoidal curve is fitted by mean of least squares to the sum-curve previously obtained. Local maxima of the sinusoid provide row centers locations. Again in images with high weed pressure this approach becomes unfeasible.

(8) Methods based on frequency analysis: Because crop rows are vertical in the $3 \mathrm{D}$ scene, they are mapped under perspective projection onto the image displaying some behavior in frequency domain. Vioix, Douzals, Truchetet, Assemat and Guillemin (2002) exploits this feature and apply a bi-dimensional Gabor filter, defined as a modulation of a Gaussian function by a cosine signal. The frequency parameter required by the Gabor filter is empirically deduced from the 2D-Fast Fourier Transform (Bossu, Gée, Guillemin, \& Truchetet, 2006). Bossu, Gée, Jones, and Truchetet (2009) apply wavelets to discriminate crop rows based on the frequency analysis. They exploit the fact that crop rows are well localized in the frequency domain; thus selecting a mother wavelet function with this frequency the crop rows can be extracted. Crops do not display clear frequency content in the Fourier space, particularly in images with a high weed pressure, therefore the application of filters based on the frequency becomes a difficult task.

\subsection{Motivational research and design of the proposed strategy}

Our work is focused on crop row detection in maize fields, for specific treatments according to the PA requirements; this requires discrimination among crops and weeds. This means that crop rows must be identified and located in the image conveniently. Some of the requirements proposed by Astrand (2005) and reported in Slaughter et al. (2008) for guidance systems can be considered for crop row detection where, in essence, the problem is similar. They are:

- Ability to locate crop rows with the maximum accuracy as possible.

- Ability to work on real-time.

- Ability to work on sown crops, not planted, which means that the time treatment and the size of the crops can vary in the field. This also means that crops and weeds have about the same size during the treatment displaying similar spectral components, i.e. discrimination between crops and weed cannot be made by size only.

- Ability to work when plants are missing in the row.

- Ability to work when there is high weed pressure.

We exploit the advantages of the existing methods introduced above and design a new strategy for crop row detection inspired on the human visual perception abilities that can cope with the above requirements, where the emphasis is put on the weed pressure treatment. This makes the main difference with respect to the above approaches, which base its design on the assumption that weed pressure is relatively low and the crop rows can be identified among crops and weeds. Moreover, because the vehicle navigates on uneven terrains with perhaps abundant irregularities, the application of geometric constraints does not suffice because the camera is continuously involved in a permanent swinging. The main contribution of the proposed method is focused on the solution of these two important problems from an automatic point of view that applies the underlying knowledge about the system and performs favorably under the above adverse conditions and also when crop plants are missing in the crop rows. Fig. 1 displays the design of the proposed approach.

The image is captured in the RGB color model. An image segmentation approach, based on vegetation index, is applied. A first image thresholding method identifies green parts (weeds/crop). By a applying a second thresholding approach, crop and weeds are separated. Based on geometrical considerations and on the knowledge about the system configuration, a template is previously built, containing those parts where crop lines are expected. By combining the information provided by this template and the crop plants, already separated after the second thresholding, crop lines are identified. By applying a total least-squares linear regression method, an adjusted line to the crop is extracted and identified by its equation.

The paper is organized as follows. Section 2 describes materials and methods used for the strategy displayed in Fig. 1. Section 3 contains results obtained by applying the proposed approach. Finally, Section 4 contains conclusions and future trends. 


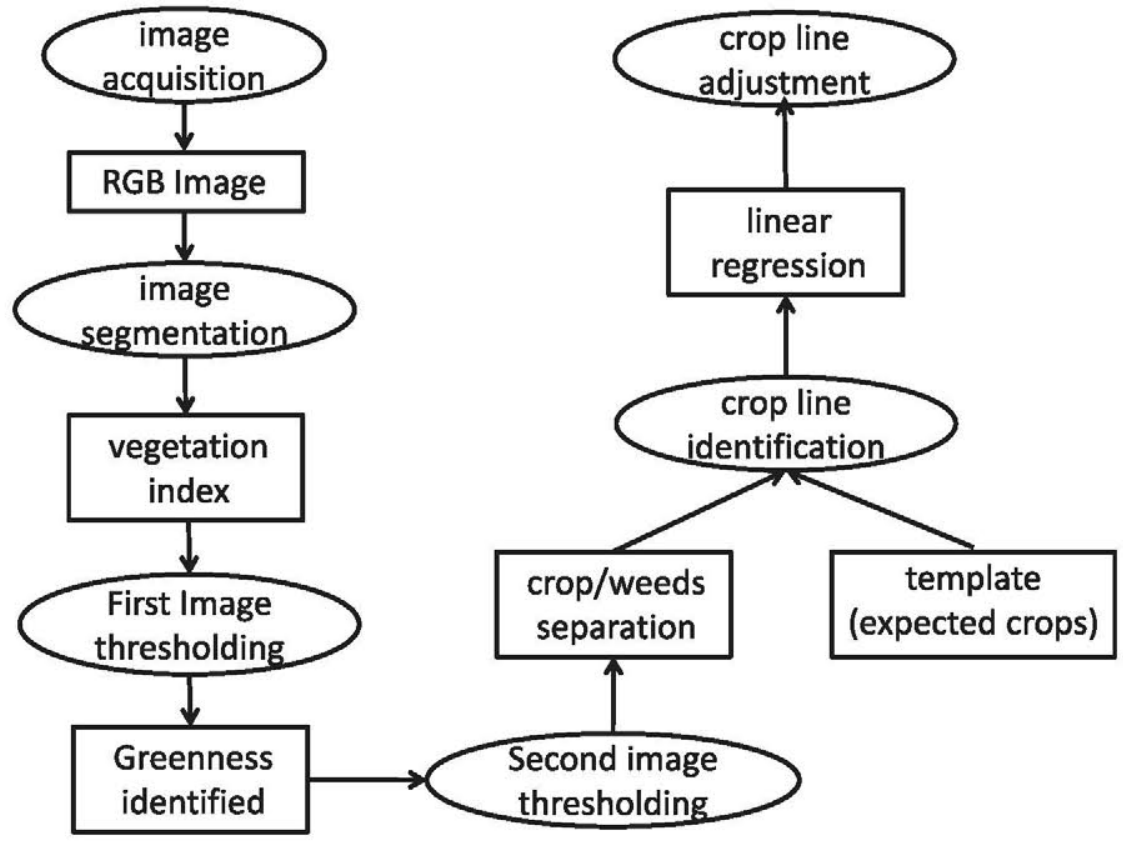

Fig. 1. Architecture and design of the proposed crop row detection approach.

\section{Materials and methods}

\subsection{Images}

All images used for this study belongs to maize crops. Two types of images have been used for image analysis. A first set of 200 real images captured with a Basler scA 1400-17fc camera during April/ May 2011, belonging to Type-1, Fig. 2(a). The digital images were captured under perspective projection and stored as 24-bit color images with resolutions of $1392 \times 1044$ pixels saved in RGB (Red, Green and Blue) color space in the TIFF format. Camera setting was: pitch and roll angles of $20^{\circ}$ and $0^{\circ}$ respectively at a height of $1.5 \mathrm{~m}$ from the ground. There were several days of acquisitions, providing some sets of images with different growth state and different conditions of illumination. They contain areas with different denseness. A second set of 100 digital images, belonging to Type-2, Fig. 2(b), captured with a Sony commercial DCR PC110E camera, with resolution of $720 \times 576$ pixels during April/May 2007 and saved also in RGB color space in the JPEG format. All were acquired in a 1.7-ha experimental field of maize on La Poveda Research Station, Arganda del Rey, Madrid. Camera setting for this type was: pitch and roll angles of $10^{\circ}$ and $0^{\circ}$ respectively and at a height of $1.5 \mathrm{~m}$ from the ground.

All methods were implemented in $\mathrm{C}++$ under the IDE Visual Studio 2008 and using the free computer vision library OpenCV (2011).

Fig. 2(a) and (b) displays two illustrative examples of both types of images, identified as Type- 1 and Type- 2 respectively. Both contain high weed densities, but the weed pressure in (b) is exceptionally high.

\subsection{Image acquisition and segmentation}

Once the image is captured in the RGB color model, the first step consists in the plant extraction or greenness image segmentation. A pixel where the predominant spectral component is the green is considered vegetation. In our images this assumption is acceptable due to the nature of the images and because they are acquired in the middle of maize fields where only soil, weed and crop plants are present. In order to discriminate between vegetation and novegetation we have used color vegetation indices (Ribeiro et al., 2005; Woebbecke et al., 1995; Xiang \& Tian, 2011), exactly the

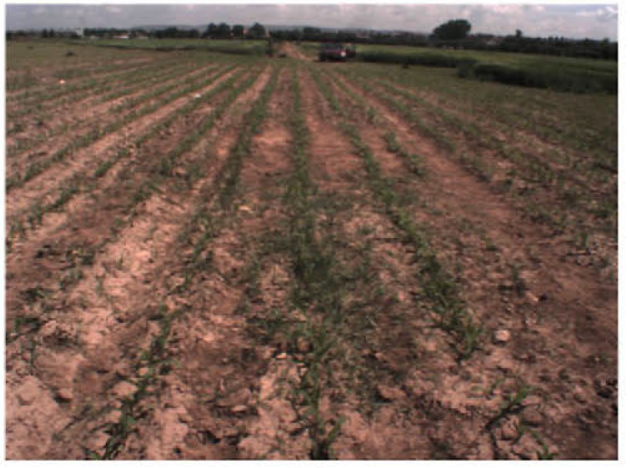

(a)

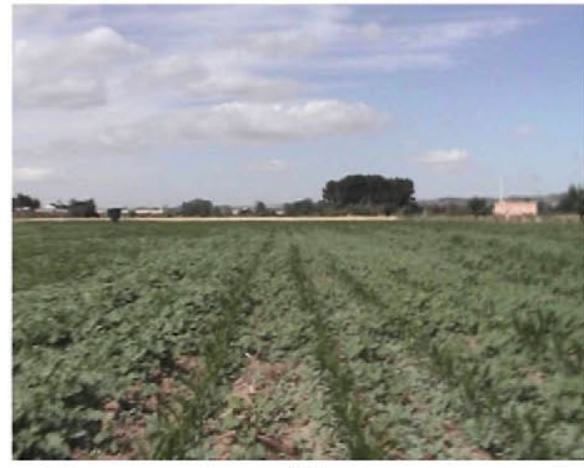

(b)

Fig. 2. Original images representing two sets of images with high weed pressure: (a) Type-1 and (b) Type-2. 
Excess Green (ExG). The choice was made after comparing the following indices and to observe how plants were identified: ExG (Meyer, Hindman, \& Lakshmi, 1998; Woebbecke et al., 1995), excess green minus excess red index or ExGR (Neto, 2004), color index of vegetation or CIVE (Kataoka, Kaneko, Okamoto, \& Hata, 2003) and NDI (Perez, Lopez, Benlloch, \& Christensen, 2000). The previous color vegetation indices (also called color indices in the literature) used are color space transformations from RGB to a space one-dimensional. Woebbecke et al. (1995) tested five indices and found the ExG index to perform well.

Next we go into details about all needed operations for extracting the ExG color index. The three spectral bands $R, G$ and $B$ are used as sources. First we normalize the values of each spectral band, this normalization is performed dividing by the highest value within spectral band for the image being analyzed. The normalized pixel values range in $[0,1]$ according to (1).

$\hat{R}=R / R_{m}, \quad \hat{G}=G / G_{m}, \quad \hat{B}=B / B_{m}$,

The actual pixel values from the images based on each $R G B$ channel are $R, G$ and $B$ for the channels red, green and blue respectively, $C_{m}$ is the highest pixel value for the channel $C$ in the image and $\hat{C}$ is the normalized pixels values for the channel $C$, where $C \in\{R, G, B\}$. The images are 24-bit color images and the pixel belongs to natural numbers set $[0,255]$.

A second normalization is performed, which is usually applied in agronomic image segmentation (Gée et al., 2008; Meyer \& Neto, 2008), with this new operation the sum of the three channels' values for a given pixel is equals to unity, as follows:

$$
r=\frac{\hat{R}}{\hat{R}+\hat{G}+\hat{B}}, \quad g=\frac{\hat{G}}{\hat{R}+\hat{G}+\hat{B}}, \quad b=\frac{\hat{B}}{\hat{R}+\hat{G}+\hat{B}}, \quad r+g+b=1 .
$$

Finally the index ExG is computed as follows:

$E x G=2 g-b-r$

Fig. 3(a) and (b) displays the ExG transformed images from the corresponding original images displayed in Fig. 2.

\subsection{Double thresholding}

In $E x G$ we can see different levels of values so that the higher the value the higher the greenness. This means that the living plant material (crop or weed) appears as white spots and the rest (soil surface, stones, shadows, straw and other debris) is black. Thus, we need an automatic approach to detect the best level separating plants from the rest. This becomes a thresholding problem. We have investigated different thresholding approaches and the adaptive Otsu's (1979) method that maximizes the interclass variance of the plant and background pixels becomes acceptable. It has been applied in different studies related to crop and weed detection (Burgos-Artizzu, Ribeiro, Guijarro, \& Pajares 2011; Jiang \& Zhao, 2010; Meyer \& Neto, 2008; Wei \& Shangfeng, 2006). It assumes bimodal histograms, representing two Gaussian intensity distributions, one of them associated to plants and the other to the rest. After this first thresholding, pixels belonging to plants are separated from the rest. Now, because plants include both weeds and crops, we can assume that even if they have similar spectral components is still possible to distinguish some spectral variation which is mapped on the ExG. Hence, we can apply a second thresholding to separate different kinds of green plants. Now, this thresholding is applied only over pixels belonging to ExG which have been identified as plants. These pixel produce a new histogram that considered as bimodal allow to apply again the Otsu's thresholding method, a second threshold is obtained that allows distinguish two different types of plants. This double thresholding approach is the key step in the proposed approach. Figs. 4 and 5(a) and (b) display the binarized images after the first and second thresholding applied to images in Fig. 3(a) and (b) respectively.

From images displayed in Fig. 4, after the first thresholding, green plants are identified and marked as white pixels, obviously displaying a high density derived from the also high weed pressure, particularly the image in Fig. 4(b). After applying the second thresholding a large number of pixels identified as weeds disappear, Fig. 5. Discussion about these results is provided in Section 3.

\subsection{Crop row identification}

After the second thresholding the next goal is to assign the corresponding straight line equation to each crop row. With such purpose we resort to methods based on linear regression where the parameters of the straight line are to be estimated from pixels considered as belonging to crop rows. At this step the problem is to determine which pixels participate in the parameter estimation for each crop row. To solve this problem, we make use of the underlying knowledge about the arrangement of crops in the maize field and also based on the geometric constraints of the camera system. They are:

(a) The number of crop rows to be detected is known.

(b) The expected location of each crop row in the image is known.

(c) The area to be explored in the image is also known and determines minimum and maximum values in the $y$-coordinates over the image.

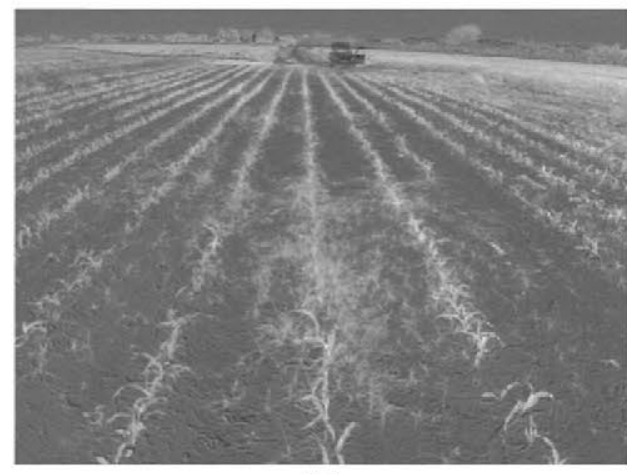

(a)

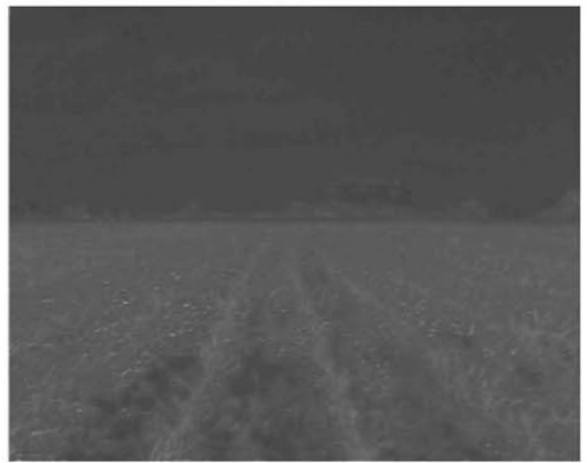

(b)

Fig. 3. Excess Green from the original images in Fig. 2: (a) Type-1 and (b) Type-2. 


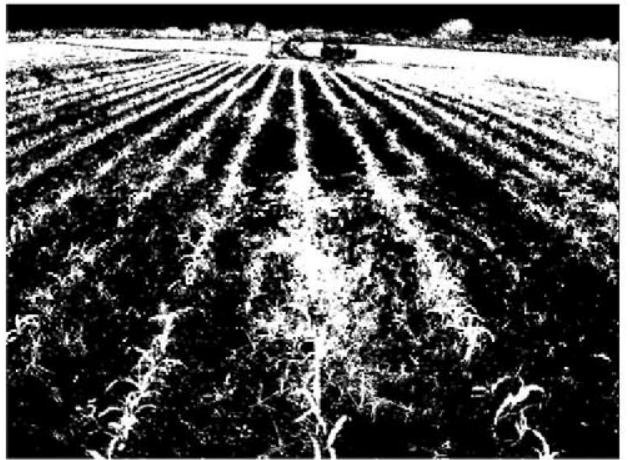

(a)

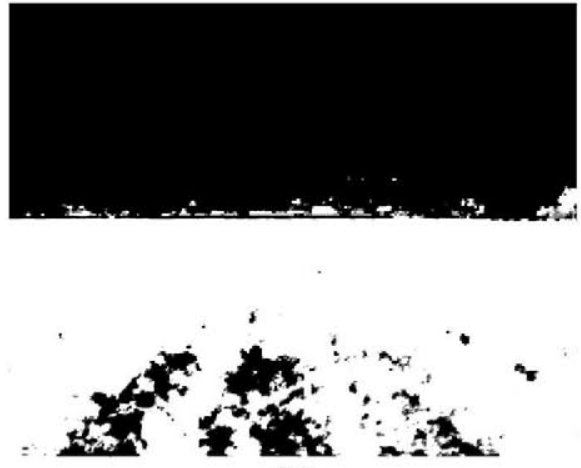

(b)

Fig. 4. Binarized images after the first thresholding based on the Otsu's method: (a) Type-1 and (b) Type-2.

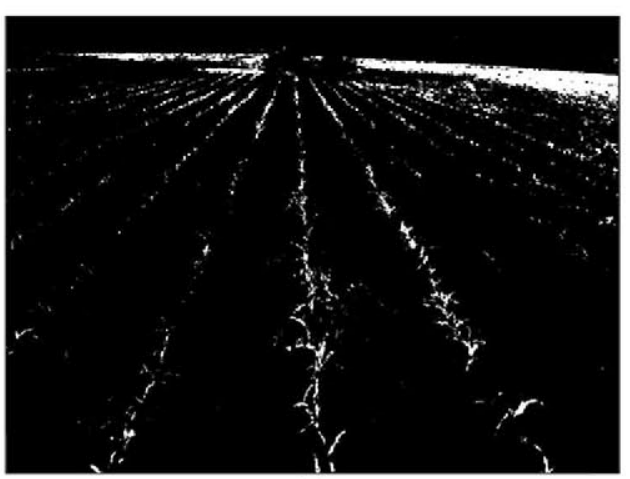

(a)

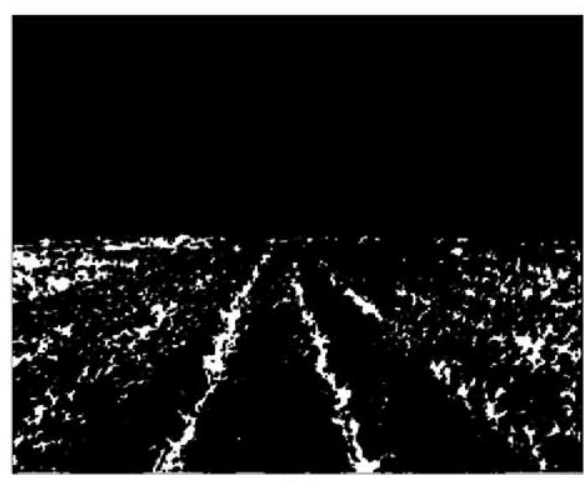

(b)

Fig. 5. Binarized images after the second thresholding based on the Otsu's method: (a) Type-1 and (b) Type-2.

On the other hand we know that the vehicle, equipped with the vision system, navigates on a real terrain presenting irregularities and roughness. This produces vibrations and also swinging mainly in the pitch and roll angles. The yaw angle is assumed to be correct because otherwise the robot will navigate erroneously and this derives in a control problem out the scope of crop row detection. Based on this fact and after capturing three video sequences consisting of 1400 images each, with the vehicle navigating at $4 \mathrm{~km} /$ $\mathrm{h}$, we have verified maximum right and left displacements around the expected crop lines in the image. Based on this fact and on the observation from the three videos, we build the corresponding template or mask to determine limits where pixels belonging to crop rows are expected. Obviously, for building these templates, we apply the knowledge about the camera settings and system geometry. Fig. 6 displays two templates for the images displayed in Fig. 5(a) and (b). They are binary images, manually built assuming previous knowledge; they contain the same number of strips than crop lines are to be estimated. Sizes of these templates are identical to the images under processing. The strips are based on the perspective projection assuming the crop rows in the field are parallel. An additional advantage of using templates is that they contain as many areas as crop lines to be detected. This means that only pixels belonging to a given area will participate on the line estimation for crop row identification.

Now, given the binary image after the second thresholding and considering the corresponding template, we apply the logical operation "and", which allows filtering those pixels that are considered as potential candidates to belong to the crop lines, i.e. pixels which participate in the estimation of the parameters involved in the straight line equations defining the crop rows. The remaining

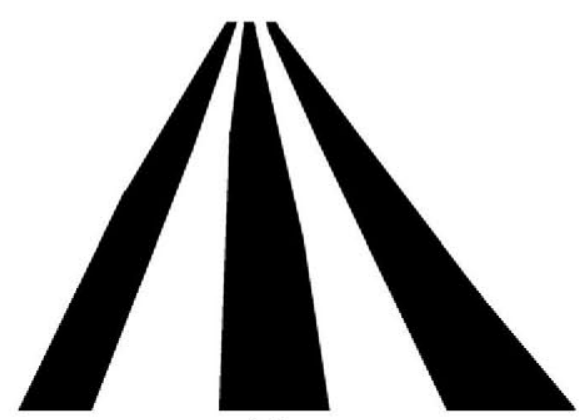

(a)

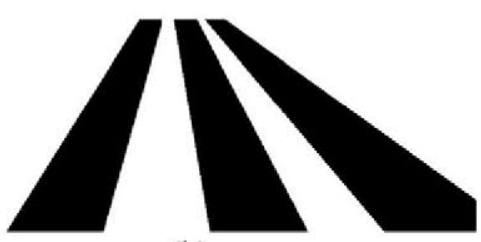

(b)

Fig. 6. Templates identifying areas where crop rows are expected: (a) for image in Fig. 5(a) and (b) for image in Fig. 5(b). 
pixels are excluded and do not participate in the parameter estimation. The use of templates allows a more accurate straight line adjustment because pixels, considered as outliers, probably belonging to weeds, are excluded from the estimation.

Now the problem has been reduced to find the best fitting straight line through a set of points. The linear least squares fitting is probably the simplest form of linear regression. Least square is a general approach to the approximate solution of over-determined systems (with more equations than unknowns). The solution minimizes the sum of the squares of the errors made in solving every single equation. The difference between an observed value and the value provided by the model is named residual. Least squares problems can be classified into linear and nonlinear depending on whether or not the residuals are linear in all unknowns. As the functions to be estimated in our approach are always straight lines, we used linear least squares, but nonlinear could be also applied (Bates \& Watts, 1988; Johnson, Correia, Yphantis, \& Halvorson, 1981; Marquardt, 1963). This choice is also justified on the basis of achieving low computational cost, making the method suitable for real-time applications. In this paper least squares is used to find the best-fitting straight line to a given set of points by minimizing the sum of the squares of the offsets ("the residuals") of the points from the straight line. The sum of the squares of the offsets is used, instead of the offset absolute values, because this allows the residuals to be treated as a continuous differentiable quantity. Outlying points can have undesirable effects on the fit due to squares of the offsets, which can be harmful. In our case, we have carried out several tests and in all of them there are not problems with results of the fitting because the use of the templates avoids the presence of outliers, as expected.

The offsets can be vertical or perpendicular with respect the straight line under estimation. With vertical offsets (classical least square) usually the errors are defined as the squared distance to fitted function from points along the vertical axis. The total leastsquares method (Golub, 1973; Golub \& Van Loan, 1980) is a natural generalization of the least-squares approximation method when the data in both $X$ and $Y$ coordinates is perturbed. For the pixels belonging to the crop row there is not an assignment of "dependent" and "independent" variables and it makes more sense to measure errors as the squared perpendicular distance to the fitted function.

Given a set of points (pixels) $X \equiv\left\{\left(x_{1}, y_{1}\right),\left(x_{2}, y_{2}\right), \ldots,\left(x_{n}, y_{n}\right)\right\}$ from the binary images after the second thresholding and belonging to an isolated zone in the image template, the goal is to estimate the best fitted straight line with equation $y=m x+a$, where the slope $m$ and the $y$-intercept $a$ are the parameters to be estimated (Markovsky \& Van Huffel, 2007). The expression to be minimized corresponds to the error or sum of offsets given by,

$E=\sum_{i=1}^{n} d_{i}=\sum_{i}^{n} \frac{\left|y_{i}-\left(a+m x_{i}\right)\right|}{\sqrt{1+m^{2}}}$,

where $d_{i}$ is the offset for the point $i$ whose coordinates are. Minimizing (4) we obtain:

$a=\frac{\sum_{i=1}^{n} y_{i}-m \sum_{i=1}^{n} x_{i}}{n}=\bar{y}-m \bar{x} ; \quad m=-B \pm \sqrt{B^{2}+1}$.

The term $B$ above is defined as follows:

$B=\frac{\left(\sum_{i=1}^{n} y_{i}^{2}-n \bar{y}^{2}\right)-\left(\sum_{i=1}^{n} x_{i}^{2}-n \bar{x}^{2}\right)}{2\left(n \bar{x} \bar{y}-\sum_{i=1}^{n} x_{i} y_{i}\right)}$.

Fig. 7 displays the final result for identification crop row for the original images of Fig. 2. We can see the straight lines, which represented the crop rows, obtained by the algorithm drawn in red on the original image. Fig. 7(a) and (b) display the crop row detected (red lines) for the images displayed in Fig. 2(a) and (b) respectively.

\section{Results}

From images displayed in Figs. 4 and 5, we can see that after the first thresholding the green plants, including crop and weeds, are recovered and identified from the original images in the corresponding images in Fig. 2. After the second thresholding, only crop plants are identified even if in the image in Fig. 2(b) where weed pressure is extremely high. This means that the proposed double Otsu strategy becomes appropriate for high weeds densities and facilitates the posterior crop row detection based on line adjustment. The performance of the double Otsu is verified for the set of images analyzed. Fig. 7 displays the three crop rows which have been identified at each image, where a straight line equation is associated to each crop row. As we can see in these images, the red lines follow the crop rows even when the crop rows appear without a symmetric distribution with respect an imaginary central axis. This is particularly appreciable in the image displayed in Fig. 7(a).

As mentioned during the introduction, the Hough transform has been applied in several methods for crop row detection, hence we compare the performance of our linear regression (OLR) approach against the Hough (HOU) transform. Both comparisons are studied after applying the double Otsu-based thresholding method. The Hough transform is implemented to work under the normal representation, polar coordinates, (Gonzalez, Woods, \& Eddins, 2009) where increment in the parameter representing the angle is set to one.

The comparison is established in terms of effectiveness and processing times or computational cost measured in time units. The effectiveness is measured based on the expert human criterion, where a line which has been detected, is considered as correct if it overlaps with the real crop row alignment according to the expert's observation. Over the set of 300 images analyzed, we compute the average percentage of correct overlapping for both OLR and HOU. Because the main goal of the proposed approach is its profit for real-time applications, we measure computational times. Also, with the goal of real-time, we have tested these performances for different image resolutions. Image resolutions differ from the ones in the original images, these resolutions have been obtained by applying a down-sampling by two to each image in both sets of available images, represented by images in Fig. 2(a) and (b) and identified as Type-1 and Type-2 respectively.

This is intended under the idea that it is possible reduce the image dimension retaining the main information without affecting the effectiveness in the crop row detection at the same time that the processing time is reduced. Table 1 displays the results. The first column contains two resolutions for each type of images (Type- 1 and Type-2). Columns second and third contain the averaged percentage of effectiveness, in terms of correct overlapping, over the sets of images studied; columns fourth and fifth display the averaged processing times, measured in milliseconds, over the sets of images analyzed. These times corresponds to the full process, i.e. image segmentation, thresholding by double Otsu and linear regression (OLR) or Hough transform (HOU).

From results in Table 1, we can infer that the best performance in terms of effectiveness is achieved by OLR with high resolution and also for images of Type-1. This is justified on the basis that images with high resolution contain more information than the ones with less resolution. With regard the type of images, it is clear that Type-2 images are much more complex than Type-1, hence the worst results obtained in terms of effectiveness for images of Type-2.

With respect the processing times, OLR outperforms HOU, this is because HOU requires more computational operations although the range of search for the angle involved in the Hough transformation 


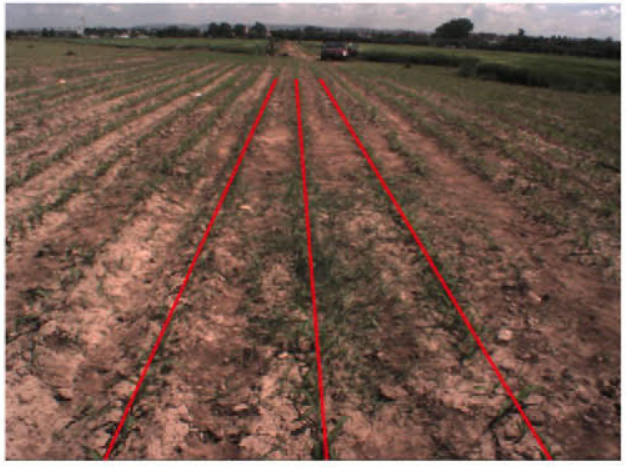

(a)

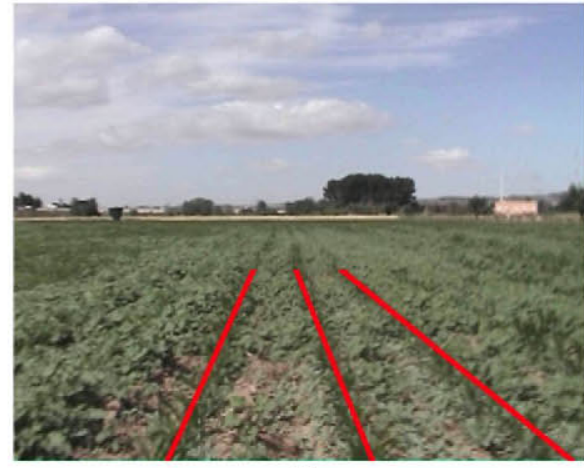

(b)

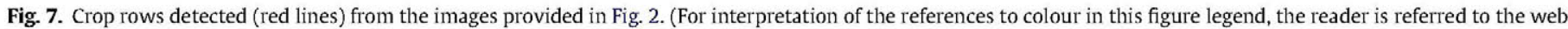
version of this article.)

Table 1

Performances of HOU and OLR approaches measured in terms of percentage of effectiveness and processing times.

\begin{tabular}{|c|c|c|c|c|}
\hline \multirow[t]{2}{*}{ Image resolution (pixels) } & \multicolumn{2}{|c|}{ Percentage of effectiveness } & \multicolumn{2}{|c|}{ Processing time (ms) } \\
\hline & HOU & OLR & HOU & OLR \\
\hline $1392 \times 1044$ (Туре- 1 ) & 89.3 & 95.5 & 4.678 & 297 \\
\hline $696 \times 522$ (Type- 1$)$ & 86.4 & 92.9 & 2.567 & 74 \\
\hline $720 \times 576$ (Type-2) & 82.4 & 89.6 & 1.935 & 81 \\
\hline $360 \times 288$ (Type-2) & 80.1 & 88.1 & 1.342 & 28 \\
\hline
\end{tabular}

was limited to range between $52^{\circ}$ and $130^{\circ}$, so only lines with the slopes defined by the expected crop lines are considered. As mentioned before, the step for indexing the angle in the cells accumulator was set to $1^{\circ}$.

Another problem concerning the Hough transform is the choice of the local maxima in the accumulator for each straight line. Indeed, the intention is to obtain a unique equation for each alignment of pixels, but in the accumulator appear different values for a unique straight line. This causes that several lines attached to each crop row are found. We can select the one with the maximum peak value in the accumulator or we can compute an averaged value among all lines involved. Both solutions often lead to errors in identifying the correct line associated to the pixels alignment. These are the main reasons justifying the outperformance of OLR against HOU.

\section{Conclusions}

We propose a method for crop row detection in maize fields with high weeds pressure. The proposed strategy is based on three main processes: image segmentation, double thresholding based on the Otsu's approach and least squares linear regression for computing equations associated to the identified crop rows. Original images are captured in the color space RGB and transformed to gray images by applying the ExG vegetation index. After this transformation, a double Otsu approach allows to separate weeds and crops, making an important contribution for this kind of images. A linear regression approach based on total least squares is applied for computing equations of straight lines associated to the crop rows. We apply the underlying knowledge about geometrical constraints embedded in the vision system to build a template that guides the process of equations computation. The performance of the proposed approach is tested with a broad set of images and the outperformance of the linear regression is compared against the classical Hough approach.

\section{Acknowledgements}

The research leading to these results has been funded by the European Union's Seventh Framework Programme [FP7/20072013] under Grant Agreement No. 245986 in the Theme NMP2009-3.4-1 (Automation and robotics for sustainable crop and forestry management). The authors wish also to acknowledge to the project AGL-2011-30442-C02-02, supported by the Ministerio de Economía y Competitividad of Spain within the Plan Nacional de $\mathrm{I}+\mathrm{D}+\mathrm{i}$.

\section{References}

Asif, M., Amir, S., Israr, A., \& Faraz, M. (2010). A vision system for autonomous weed detection robot. Int. J. Comput. Electr. Eng., 2(3), 486-491.

Astrand, B. (2005). Vision based perception or mechatronic weed control. Doctor of Philosophy Thesis, Chalmers and Halmstad Universities, Sweden.

Astrand, B., \& Baerveldt, A. J. (2005). A vision based row-following system for agricultural field machinery. Mechatronics, 15, 251-269.

Bates, D. M., \& Watts, D. G. (1988). Nonlinear regression and its applications. New York: Wiley,

Billingsley, J., \& Schoenfisch, M. (1997). The successful development of a vision guidance system for agriculture. Computers and Electronics in Agriculture, 16, 147-163.

Bossu, J., Gée, Ch., Guillemin, J. P., \& Truchetet, F. (2006). Development of methods based on double Hough transform and Gabor filtering to discriminate crop and weeds in agronomic images. In Proc. SPIE 1 8th annual symposium electronic imaging science and technology, paper No. 23, San Jose, USA (Vol. 6070).

Bossu, J., Gée, Ch., Jones, G., \& Truchetet, F. (2009). Wavelet transform to discriminate between crop and weed in perspective agronomic images. Computers and Electronics in Agriculture, 65, 133-143.

Burgos-Artizzu, X. P., Ribeiro, A., Guijarro, M., \& Pajares, G. (2011). Real-time image processing for crop/weed discrimination in maize fields. Computers and Electronics in Agriculture, 75(2), 337-346.

Burgos-Artizzu, X. P., Ribeiro, A., Tellaeche, A., Pajares, G., \& Fernández-Quintanilla, C. (2009). Improving weed pressure assessment using digital images from an experience-based reasoning approach. Computers and Electronics in Agriculture, $65,176-185$.

Davies, G., Casady, W., \& Massey, R. (1998). Precision agriculture: an introduction. Water Quality Focus Guide WQ450. Available from: <http:// extension.missouri.edu/explorepdf/envqual/wq0450.pdf>. 
Fontaine, V., \& Crowe, T. G. (2006). Development of line-detection algorithms for local positioning in densely seeded crops. Canadian Biosystems Engineering, $48(7), 19-29$.

Gée, Ch., Bossu, J., Jones, G., \& Truchetet, F. (2008). Crop/weed discrimination in perspective agronomic images. Computers and Electronics in Agriculture, 60, 49-59.

Golub, G. (1973). Some modified matrix eigenvalue problems. SIAM Review, 15 , $318-344$

Golub, G., \& Van Loan, C. (1980). An analysis of the total least squares problem. SIAM Journal on Numerical Analysis, 17, 883-893.

Gonzalez, R. C., Woods, R. E., \& Eddins, S. L. (2009). Digital image processing using $M A T L A B$. Upper Saddle River, NI: Prentice Hall.

Hague, T., Marchant, J. A., \& Tillett, D. (1997). A system for plant scale husbandry. Precision Agriculture, 635-642.

Hague, T., Tillet, N., \& Wheeler, H. (2006). Automated crop and weed monitoring in widely spaced cereals. Precision Agriculture, 1(1), 95-113.

Hough, P. V. C. (1962). A method and means for recognizing complex patterns. US Patent Office No. 3069654

Ji, R., \& Oi, L. (2011). Crop-row detection algorithm based on random Hough transformation. Mathematical and Computer Modelling, 54, 1016-1020.

Jiang, G., \& Zhao, C. (2010). A vision system based crop rows for agricultural mobile robot. In International conference on computer application and system modeling (ICCASM) (Vol. 11, pp.V11-142-V11-145, 22-24).

Johnson, M. L., Correia, J. J., Yphantis, D. A., \& Halvorson, H. R. (1981). Analysis of data from the analytical ultracentrifuge by nonlinear least-squares techniques. Biophysical Journal, 36(3), 575-588.

Jones, G., Gée, Ch., \& Truchetet, F. (2009a). Modelling agronomic images for weed detection and comparison of crop/weed discrimination algorithm performance. Precision Agriculture, 10,1-15.

Jones, G., Gée, Ch., \& Truchetet, F. (2009b). Assessment of an inter-row weed infestation rate on simulated agronomic images. Computers and Electronics in Agriculture, 67, 43-50.

Kataoka, T., Kaneko, T. Okamoto, H. \& Hata, S. (2003). Crop growth estimation system using machine vision. In The 2003 IEEE/ASME international conference on advanced intelligent mechatronics.

Kise, M., \& Zhang, Q. (2008). Development of a stereovision sensing system for 3D crop row structure mapping and tractor guidance. Biosystems Engineering, 101. 191-198.

Kise, M., Zhang, Q., \& Rovira-Mâs, F. (2005). A stereovision-based crop row detection method for tractor-automated guidance. Biosystems Engineering, 90(4), 357-367.

Leemans, V., \& Destain, M. F. (2006). Application of the Hough transform for seed row location using machine vision. Biosystems Engineering, 94(3), 325-336.

Marchant, J. (1996). Tracking of row structure in three crops using image analysis. Computers and Electronics in Agriculture, 15, 161-179.

Markovsky, I., \& Van Huffel, S. (2007). Overview of total least-squares methods. Signal Processing, 87(10), 2283-2302.

Marquardt, D. W. (1963). An algorithm for least-squares estimation of nonlinear parameters. Journal of the Society for Industrial and Applied Mathematics, 11(2), 431-441.

Meyer, G. E., Hindman, T. W., \& Lakshmi, K. (1998). Machine vision detection parameters for plant species identification. Bellingham, WA: SPIE

Meyer, G. E. \& Neto, J. C. (2008). Verification of color vegetation indices for automated crop imaging applications. Computers and Electronics in Agriculture, $63,282-293$
Neto, J. C. (2004). A combined statistical-soft computing approach for classification and mapping weed species in minimum tillage systems. Lincoln. NE: University of Nebraska.

Olsen, H. J. (1995). Determination of row position in small-grain crops by analysis of video images. Computers and Electronics in Agriculture, 12, 147-162.

Onyango, C. M., \& Marchant, J. A. (2003). Segmentation of row crop plants from weeds using colour and morphology. Computers and Electronics in Agriculture, 39, 141-155.

OpenCV (2011). http://opencv.willowgarage.com/wiki/ 26.10.11.

Otsu, N. (1979). A threshold selection method from gray-level histogram. IEEE Transactions on Systems Man and Cybernetics, 9, 62-66.

Perez, A. J., Lopez, F., Benlloch, J. V., \& Christensen, S. (2000). Color and shape analysis techniques for weed detection in cereal fields. Computer and Electronics in Agriculture, 25, 197-212.

Pla, F., Sanchiz, J. M., Marchant, J. A., \& Brivot, R. (1997). Building perspective models to guide a row crop navigation vehicle. Image and Vision Computing, 15, 465-473.

Ribeiro, A., Fernández-Quintanilla, C., Barroso, J., \& García-Alegre, M. C. (2005). Development of an image analysis system for estimation of weed. In Proceedings of the 5th European conference on precision agriculture (5ECPA) (pp. 169-174).

Rovita-Más, F., Zhang, Q., \& Reid, J. F. (2008). Stereo vision three-dimensional terrain maps for precision agriculture. Computers and Electronics in Agriculture, 60, 133-143.

Rovira-Más, F., Zhang, Q., Reid, J. F., \& Will, J. D. (2005). Hough-transform-based vision algorithm for crop row detection of an automated agricultural vehicle. Journal Automobile Engineering, 219, 999-1010. Part D.

Sainz-Costa, N., Ribeiro, A., Burgos-Artizzu, X., Guijarro, M., \& Pajares, G. (2011). Mapping wide row crops with video sequences acquired from a tractor moving at treatment speed. Sensors, 11, 7095-7109.

Slaughter, D. C., Giles, D. K., \& Downey, D. (2008). Autonomous robotic weed control systems: A review. Computers and Electronics in Agriculture, 61, 63-78.

Søgaard, H. T., \& Olsen, H. J. (2003). Determination of crop rows by image analysis without segmentation. Computers and Electronics in Agriculture, 38, 141-158.

Tellaeche, A., Burgos-Artizzu, X. P., Pajares, G., \& Ribeiro, A. (2008b). A vision-based method for weeds identification through the Bayesian decision theory. Pattern Recognition, 41, 521-530.

Tellaeche, A., Burgos-Artizzu, X., Pajares, G., Ribeiro, A., \& Fernăndez-Quintanilla, C. (2008a). A new vision-based approach to differential spraying in precision agriculture. Computers and Electronics in Agriculture, 60(2), 144-155.

Tellaeche, A., Pajares, G., Burgos-Artizzu, X. P., \& Ribeiro, A. (2011). A computer vision approach for weeds identification through support vector machines. Applied Soft Computing, 11, 908-915.

Vioix, J. B., Douzals, J. P., Truchetet, F., Assemat, L., \& Guillemin, J. P. (2002). Spatial and spectral method for weeds detection and localization. EURASIP JASP, 7 $679-685$.

Wei, Z., \& Shangfeng, D. (2006). Machine vision recognizing position baseline in cropland. Journal of China Agricultural University, 11(4)

Woebbecke, D. M., Meyer, G. E., von Bargen, K., \& Mortensen, D. A. (1995). Shape features for identifying young weeds using image analysis. Transactions on American Society of Agricultural Engineering, 38(1), 271-281.

Xiang, H., \& Tian, L. (2011). An automated stand-alone in-field remote sensing system (SIRSS) for in-season crop monitoring. Computers and Electronics in Agriculture, 78(1), 1-8. ISSN 0168-1699. 